\title{
Node Layout of Recycling Network for Cell Phones in China under the Third Party Logistics
}

\author{
Danning $\mathrm{Li}^{1}$, Xianliang Shi ${ }^{1}$ and Chunhua $\mathrm{Shi}^{2}$ \\ ${ }^{1}$ School of Economics and Management, Beijing Jiaotong University, Beijing, \\ China, 100044 \\ ${ }^{2}$ Higher Education Press, Beijing, China, 100029 \\ 13120591@bjtu.edu.cn; xlshi@bjtu.edu.cn; shichh@hep.com.cn
}

\begin{abstract}
With the increasing acceleration of updating rate for mobile phones in China, the processing procedure of cell phones has aroused a problem for the whole society. Meantime, environmental pollution and waste of resources result from improper processing approaches of such products. In this paper, the processing modes and corresponding reverse logistics network are analyzed scientifically and completely in terms of recycling by the third party reverse logistics enterprises. Then, a mathematical programming model is proposed in the case of multi-varieties phones and stochastic circumstances to handle the problem of node arrangement and facility location, which taking both cost objective and environment objective into consideration. To combat the model, we design a hybrid intelligent algorithm consisting of genetic algorithm, stochastic simulation and linear programming. Finally a numerical example validates the feasibility of the model and sensitivity analysis is conducted to illustrate its reliability. Such method can be treated as a practice guideline and some suggestions regarding the layout construction are put forward in the end.
\end{abstract}

Keywords: The third party reverses logistics, node layout, stochastic circumstances, multi-objective programming, hybrid intelligent algorithm

\section{Introduction}

In the mobile phone industry, smart phones account for an increasing percentage of the whole market (Figure 1) throughout China. And cell phones are remarkably refreshed more and more fast with the advent of $3 \mathrm{G}$ and $4 \mathrm{G}$ network, thus resulting in the disposal of mobile phones which have been replaced or updated. According to statistics, there were more than 1.1 billion phone users throughout China, and as many as 3 hundred million cell phones which were in the idle state in China in 2013, and the number of used batteries was higher than 5 hundred million. At the same time, the coefficient of recovery was less than $1 \%$. All throws severe challenges to phone industry. According to researchers, used phones after being tested can be sold on secondary market. Besides, waste phones can be changed into things of values because of circuit boards, precious metals such as gold, silver, nickel and chromium they contained. For these phones, improper ways of handling and deficiency of recycling processes not only cause air pollution and water pollution, but also contribute to waste of resources. In China, adverse effects caused by mobile phones are even more serious because of the imperfect recycling system and unsmooth reverse logistics channels. Based on references, if waste components and parts flow into landfills with household refuse, heavy metal ions can lead to water pollution and cadmium in one battery can pollute 60 thousand liters water. If they are under incineration disposal, heavy metal ions will be oxidized and result in atmospheric contamination, which poses a threat to human health. 


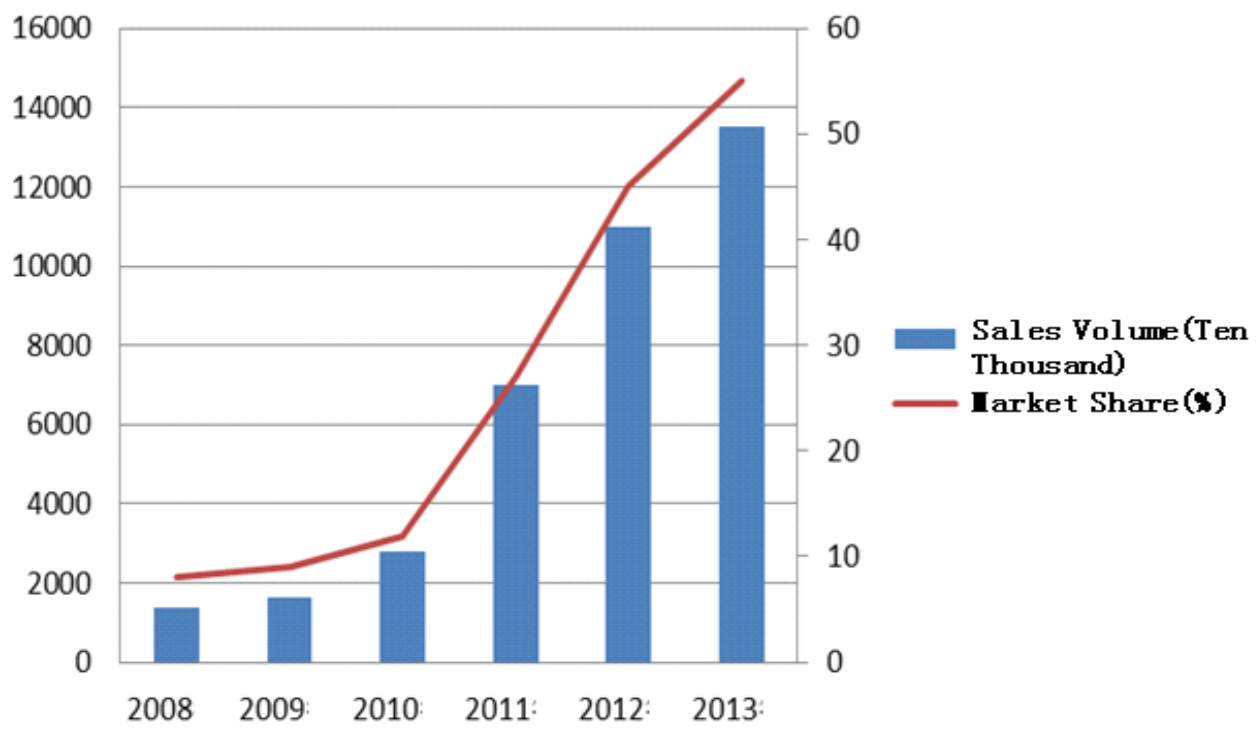

Figure 1. Development of Smart Phones in China

Based on Extended Producer Responsibility (EPR), many mobile phone manufacturers have begun to explore ways of recycling their products. Accordingly, the government affords them a certain amount of policy and financial support. Such circumstance provides an opportunity to the development of professional third party reverse logistics companies. These companies can not only reduce the fixed assets investment and operations management cost of manufacturers, but also predict the number of recycling effectively and use shared services to attain economies of scale. So in this paper, we analyze and design the reverse logistics network for mobile phones from the perspective of the third party reverse logistics enterprises.

\section{Literature Review}

Guiltinan and Nwokoye [1] first analyzed the network structure of reverse logistics system qualitatively. Ginter and Starling [2], and Batta and Chiu [3] made further research on the characteristics of reverse logistics network respectively. For the design of separate reverse logistics network, Jayarama, et al., [4] formulated a mixed integer programming model to determine the number and location of facilities in the network by analyzing technical operational processes of an electronic equipment remanufacturing enterprise and integrating its costs of various aspects. Fleischmann, et al., [5] proposed a recycling logistics network model based on single product and unlimited capacity, and he applied it into duplicating machine remanufacturing. Bautista and Pereria [6] analyzed the municipal waste management and established the relationship between the set covering problem and the MAX-SAT problem directed at facility location, and then developed a genetic algorithm and a GRASP heuristic respectively to solve each formulation. Langella [7] came up with a dynamic mixed-integer linear programming model to settle network design problem under certain circumstances and used heuristics to tackle it. Pati, et al., [8] formulated a mixed integer goal programming model integrating cost, product quality and environmental benefits to make contributions to paper recycling logistics system. Alumur, et al., [9] considered a multi-period setting with limited capacity and conducted parametric and scenario analysis. Eskandarpour, et al., [10] constructed a multi-objective model and used a parallel multi-objective heuristic based on variable neighborhood search to solve it. Harris and Martin [11] investigated the evolution and related issues of reverse logistics aimed online retailing. As for aspect of the third party and cell phones, Yun [12] analyzed the network framework of recycling and re-manufacturing under third 
party. Then a two-period recycling method was proposed to achieve optimal profit. Govindan, et al., [13] used interpretive structural modeling to combat selection of third party reverse logistics provider after dissecting the whole process. Mou, et al., [14] used a variant Weber-triangle model taking into account various costs and industrial accumulation effect to make location decisions for e-waste. And a waste printed circuit boards recycling industry is discussed as a case study. Hou, et al., [15] took up analytic network process to analyze three modes for recycling cell phones. That is outsourcing mode, joint business mode and self-supporting mode. And outsourcing is proved to be the best. As can be seen, most problems and methods discussed in the existing literature are raised under certainty. And the minimum total cost is always the single goal. What's more, the structural designs for detachable remanufacturing products are oversimplified and don't carefully consider characters of mobile phones. As the third party has various advantages taking in charge of recycling, such industry should make full use of this mode. So in this paper, we first comprehensively analyze the reverse logistics processes of cell phones. And then we establish stochastic programming model under uncertainty aimed at multi products. The objective function contains environmental goal as well as cost objective. Finally we design a hybrid intelligent algorithm to tackle such issue.

\section{Network Structure and Process Design}

For mobile phones, compared with other household appliances, small volume, high value, low retail and display cost and cash spot result in low degree of concentration in retailers. So manufacturers can take full advantage of retailers to recycle phones from customers. Meanwhile, their life cycles are often short with the increasing ratio of updating, which offers the possibility for economies of scale.

Generally reverse logistics is characterized by complexity and uncertainty. Because mobile phones are technology-intensive products with various models, types and configurations, and the aging degrees and causes of recycling are different, the process of recycling needs multiple industries, technical means, capital investment and advanced equipment, which puts forward lots of requirements to the construction of network. From the perspective of the third party enterprises, they should mainly consider three stages when constructing reverse logistics network. First, recycle old products from users by agencies or through retail outlets. Second, send the initial recycling products to disassembling and processing centers via the third party warehouses. Third, make final disposition decisions according to the consequence from disassembling and processing centers.

According to the state quote for the sales and recycling of mobile phones in China, during the initial stage of construction, a reverse logistics network based on the EPR should make appropriate treatments in accordance with the mobile phones' states. Figure 2 displays the reverse logistics network of mobile phones in general $[16,17]$.

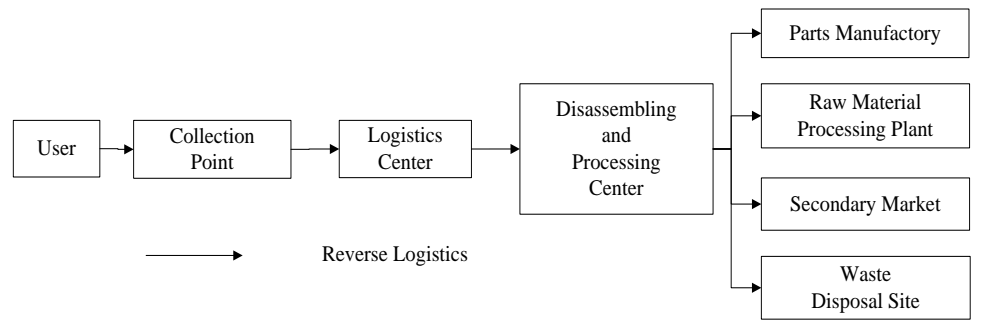

Figure 2. Reverse Logistics Network

Collection Point: provide service of old mobile phone recycling. Customers can send their phones to retail outlets (including telecom operators), communities, maintenance 
points, etc. To keep things simple in this article, we only take retail outlets as collection points.

Logistics Center: finish collecting mobile phones in a certain region and provide temporary storage service and simple classification service.

Disassembling and Processing Center: equipped with appropriate equipment. Make further decisions for mobile phones after detection / classification, repair / renovation, disassembling, smashing and other operations. Parts which have remanufacturing or reuse value will be sent to parts manufactories. Raw materials and products that have resale value will be sent to processing plants and secondary markets respectively. Waste produced from such processes will be transported to waste disposal sites where it can get harmless treatments in case it causes pollution and secondary contamination. Figure 3 illustrates the process strategies.

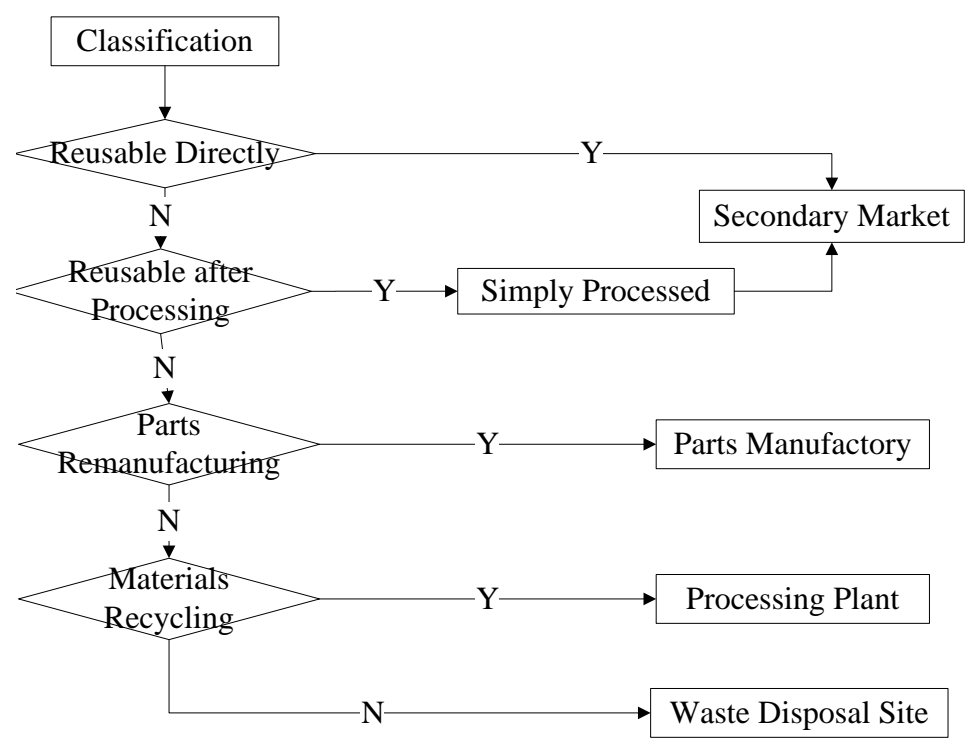

Figure 3. Diagram Graph of Reverse Process Strategies

\section{Model Construction}

\subsection{Problem Statement and Model Assumptions}

In the paper, we consider establishing a reverse logistics network model basically based on the recycling of the third party for China. For a third party reverse logistics enterprise, it needs to transport mobile phones recycled from collection points to disassembling and processing centers via logistics centers, and complete tasks in corresponding facilities. Locations of collection points, parts manufactories, raw material processing plants, secondary markets and waste disposal sites are given and determined. What have to be solved are the site selections of logistics centers and disassembling and processing centers, and reasonable flow distributions of reverse logistics quantities. For this complex multi-level location problem, we set up a mathematic model under uncertainty in order to take quantitative analyses of location economics. In this model, we take fixed asset investment costs, transportation costs and operating costs into account and take the minimum total cost as an objective function. In addition, though we recycle old phones out of protection of the environment, the recycling processes and establishments and operations of disassembling and processing centers may have negative effects on environment and life of citizens. So setting a single goal goes against our 
original intention. And in the paper, we bring in the environmental goal in terms of the public and construct multi-objective model to generate strategies.

Assumption 1: consider single period and static model; each quantity of phones recycled in each collection point is a random variable which obeys a given distribution and these random variables are mutually independent. Remanufacturing rate, raw material processing rate, resale rate and reject rate are certain.

Assumption 2: the number and the location of each type of facilities are given, and we have to screen facilities in this specified range.

Assumption 3: transportation cost is directly proportional to transportation quantity and distance; warehouse costs (coupled with handling costs) are considered together with operating costs and will not be calculated separately.

Assumption 4: only capacities of logistics centers and disassembling and processing centers are limited, and the capacities are given and certain.

\subsection{Notations}

Subscripts:

$$
\begin{aligned}
& j \_ \text {type of products }(j=1,2, \cdots, J) \\
& i \_ \text {collection point }(i=1,2, \cdots, I) \\
& l_{\_} \text {alternative logistics center }(l=1,2, \cdots, L) \\
& h \_ \text {alternative disassembling and processing center }(h=1,2, \cdots, H) \\
& a \_ \text {parts manufactory }(a=1,2, \cdots, A) \\
& b \_ \text {raw material processing plant }(b=1,2, \cdots, B) \\
& e_{\longrightarrow} \text { secondary market }(e=1,2, \cdots, E) \\
& d \_ \text {waste disposal site }(d=1,2, \cdots, D)
\end{aligned}
$$

Parameters:

$Q_{i j}-$ amount of product $j$ recycled from collection point $i$;

$\alpha_{1 j}, \alpha_{2 j}, \alpha_{3 j}, \alpha_{4 j}$ remanufacturing rate, raw material processing rate, resale rate and reject rate of product $j$;

$c_{i l}^{0}, c_{l h}^{1}, c_{h a}^{2}, c_{h b}^{3}, c_{h e}^{4}, c_{h d}^{5}, c_{i h}^{6}$ - distance between each facility;

$T C —$ transportation cost per unit weight per unit distance;

$r_{l j}^{0}, r_{h j}^{1} \longrightarrow$ unit operating cost for product $j$ in logistics center $l$ and disassembling and processing center $h$ respectively;

$F_{l}^{0}, F_{h}^{1}$ — fixed asset investment costs for logistics center $l$ and disassembling and processing center $h$ respectively;

$M_{l}^{0}, M_{h}^{1} \longrightarrow$ maximum processing capacities for logistics center $l$ and disassembling and processing center $h$ respectively;

$N_{l}, N_{h}$ number limitations of logistics centers and disassembling and processing centers;

$G_{j} \longrightarrow$ weight of product $j$;

Decision variables:

$q_{i l j}^{0}, q_{l h j}^{1}, q_{h a j}^{2}, q_{h b j}^{3}, q_{h e j}^{4}, q_{h d j}^{5}-$ volume of product $j$ between each facility;

$X_{l}^{0}, X_{h}^{1} \longrightarrow 0-1$ variables which represent if logistics center $l$ and disassembling and processing center $h$ are selected respectively. 


\subsection{Objective Function}

Cost objective. From the perspective of an enterprise, overall cost for starting and operating a business is an important element when considering at first.

$$
\begin{aligned}
& \min Z_{1}=\sum_{l} \sum_{j} \sum_{i} q_{i l j}^{0} \cdot r_{l j}^{0}+\sum_{h} \sum_{j} \sum_{l} q_{l h j}^{1} \cdot r_{h j}^{1} \\
& +\sum_{l} F_{l}^{0} \cdot X_{l}^{0}+\sum_{h} F_{h}^{1} \cdot X_{h}^{1} \\
& +\sum_{j} \sum_{l} \sum_{i} q_{i l j}^{0} \cdot c_{i l}^{0} \cdot G_{j} \cdot T C+\sum_{j} \sum_{h} \sum_{l} q_{l h j}^{1} \cdot c_{l h}^{1} \cdot G_{j} \cdot T C+\sum_{j} \sum_{a} \sum_{h} q_{h a j}^{2} \cdot c_{h a}^{2} \cdot G_{j} \cdot T C \\
& +\sum_{j} \sum_{b} \sum_{h} q_{h b j}^{3} \cdot c_{h b}^{3} \cdot G_{j} \cdot T C+\sum_{j} \sum_{e} \sum_{h} q_{h e j}^{4} \cdot c_{h e}^{4} \cdot G_{j} \cdot T C+\sum_{j} \sum_{d} \sum_{h} q_{h d j}^{5} \cdot c_{h d}^{5} \cdot G_{j} \cdot T C
\end{aligned}
$$

This objective function stands for minimizing total cost. The first two items mean operating costs. The third and fourth express fixed asset investment costs. The last six items represent transportation costs.

Environment objective. As what has been discussed in article [18], a responsive network for after-sale services of high-tech products contains several objectives. A reverse logistics is planned for the sake of the whole society. Apart from cost, environment objective should be taken into account when setting up the network. Only by cutting down the effect on the environment can the whole network operate smoothly and effectively in the long run.

$$
\operatorname{minZ}_{2}=\gamma \sum_{h} \sum_{i} \frac{\sum_{j} \sum_{l} q_{l h j}^{1}}{c_{i h}^{6}}
$$

This objective function means minimizing environmental effects on consumption regions caused by disassembling and processing centers. Such effects are directly proportional to the number of products disposed in the center, while are inversely proportional to distance between the center and consumption region (collection point). $\gamma$ is a coefficient.

\subsection{Constraint Conditions}

$$
\begin{aligned}
& Q_{i j}=\sum_{l} q_{i l j}^{0}(\forall i \in I, j \in J) \\
& \sum_{i} q_{i l j}^{0}=\sum_{h} q_{l h j}^{1}(\forall l \in L, j \in J) \\
& \alpha_{1 j} \sum_{l} q_{l h j}^{1}=\sum_{a} q_{h a j}^{2}(\forall h \in H, j \in J) \\
& \alpha_{2 j} \sum_{l} q_{l h j}^{1}=\sum_{b} q_{h b j}^{3}(\forall h \in H, j \in J) \\
& \alpha_{3 j} \sum_{l} q_{l h j}^{1}=\sum_{e} q_{h e j}^{4}(\forall h \in H, j \in J) \\
& \alpha_{4 j} \sum_{l} q_{l h j}^{1}=\sum_{d} q_{h d j}^{5}(\forall h \in H, j \in J) \\
& \alpha_{1 j}+\alpha_{2 j}+\alpha_{3 j}+\alpha_{4 j}=1(\forall j) \\
& \sum_{i} \sum_{j} q_{i l j}^{0} \leq M_{l}^{0} \cdot X_{l}^{0}(\forall l \in L) \\
& \sum_{l} \sum_{j} q_{l h j}^{1} \leq M_{h}^{1} \cdot X_{h}^{1}(\forall h \in H)
\end{aligned}
$$




$$
\begin{gathered}
\sum_{l} X_{l}^{0} \leq N_{l} \\
\sum_{h} X_{h}^{1} \leq N_{h} \\
q_{i l j}^{0}, q_{l h j}^{1}, q_{h a j}^{2}, q_{h b j}^{3}, q_{h e j}^{4}, q_{h d j}^{5} \geq 0(j \in J, i \in I, l \in L, h \in H, a \in A, b \in B, d \in D, e \in E) \\
X_{l}^{0}, X_{h}^{1}=0 \operatorname{or} 1(l \in L, h \in H)
\end{gathered}
$$

Equation (1) illustrates that products of type recycled in collection point are transported to logistics centers entirely. Equation (2) ensures that input and output of each logistics center are equal. Equation (3) shows all products in disassembling and processing centers will be sent to parts manufactories if the parts can be remanufactured. The same is true of (4-6). Equation (7) ensures input of each disassembling and processing center are the same with its output. Inequalities $(8,9)$ mean capacity limitations. Inequalities $(10,11)$ mean number limitations.

\subsection{Transformation of Objective Function}

For the two objectives in this model, though they belong to different categories and dimensions are different, they are both linear functions about decision variables. According to literature [19], we reinvest objective function $Z_{2}$ with coefficient $\beta$. Compared with objective function $Z_{1}, \beta$ can be understood as the cost for processing unit environmental effect caused by facilities. Then we construct final objective function $\min Z=Z_{1}+\beta Z_{2}$ and transform former bi-objective problem to a single objective problem.

\subsection{Model Processing}

For models that have random parameters, when processing we have to transform them into expected value models first, and the constraint conditions keep unchanged. The transformed model is as follows:

$$
\begin{aligned}
& \min E(Z)=E\left[\sum_{l} \sum_{j} \sum_{i} q_{i l j}^{0} \cdot r_{l j}^{0}+\sum_{h} \sum_{j} \sum_{l} q_{l h j}^{1} \cdot r_{h j}^{1}+\sum_{l} F_{l}^{0} \cdot X_{l}^{0}+\sum_{h} F_{h}^{1} \cdot X_{h}^{1}\right. \\
& +\sum_{j} \sum_{l} \sum_{i} q_{i l j}^{0} \cdot c_{i l}^{0} \cdot G_{j} \cdot T C+\sum_{j} \sum_{h} \sum_{l} q_{l h j}^{1} \cdot c_{l h}^{1} \cdot G_{j} \cdot T C+\sum_{j} \sum_{a} \sum_{h} q_{h a j}^{2} \cdot c_{h a}^{2} \cdot G_{j} \cdot T C \\
& +\sum_{j} \sum_{b} \sum_{h} q_{h b j}^{3} \cdot c_{h b}^{3} \cdot G_{j} \cdot T C+\sum_{j} \sum_{e} \sum_{h} q_{h e j}^{4} \cdot c_{h e}^{4} \cdot G_{j} \cdot T C+\sum_{j} \sum_{d} \sum_{h} q_{h d j}^{5} \cdot c_{h d}^{5} \cdot G_{j} \cdot T C \\
& \left.+\beta \gamma \sum_{h} \sum_{i} \frac{\sum_{j} \sum_{l} q_{l h j}^{1}}{c_{i h}^{6}}\right]
\end{aligned}
$$

When solving expected value models, traditional way is converting them into deterministic models, which is simple and effective. But it also has its disadvantages. That is to say, taking the average value instead of parameters themselves ignores their distribution information [20] during the process. We cannot reflect random fluctuation of parameters when calculating, thus offering an upper bound of the objective function as a result [21]. So in some cases, what we get is not that satisfying [22]. In this paper, we adopt hybrid intelligent algorithm to tackle this problem [23]. We mainly use genetic algorithm coupled with the other two approaches. When calculating the value of objective function, we simulate the uncertain function through way of stochastic simulation. Additionally, linear programming is used to solve the optimization problem above and get 
the value of the objective function. The processes of stochastic simulation and linear programming are as follows.

$Q_{i j}$ (The amount of product $j$ recycled from collection points $i$ ) is a random variable defined in the probability space $(\Omega, A, \operatorname{Pr})$. So the whole amounts can be defined as a random vector $Q=\left(Q_{11}, \cdots Q_{I J}\right)$ in the probability space $(\Omega, A, \operatorname{Pr})$. For each $\eta \in \Omega$, $Q(\eta)$ is the realized value of recycling vector? So the feasible solution set of facility selection $\quad\left(X^{0}, X^{1}\right) \quad$ is $S(\eta)=\left\{q_{i l j}^{0}, q_{l h j}^{1}, q_{h a j}^{2}, q_{h b j}^{3}, q_{h e j}^{4}, q_{h d j}^{5} \mid\right.$ s.t.(1) (9),(11) $\} \quad$. For each $\eta \in \Omega$, the minimal total cost is decided by (15).

$$
\begin{aligned}
& C\left(X^{0}, X^{1} \mid \eta\right)=\min _{s \in S(\eta)} Z=\sum_{l} \sum_{j} \sum_{i} q_{i l j}^{0} \cdot r_{l j}^{0}+\sum_{h} \sum_{j} \sum_{l} q_{l h j}^{1} \cdot r_{h j}^{1} \\
& +\sum_{j} \sum_{l} \sum_{i} q_{i l j}^{0} \cdot c_{i l}^{0} \cdot G_{j} \cdot T C+\sum_{j} \sum_{h} \sum_{l} q_{l h j}^{1} \cdot c_{l h}^{1} \cdot G_{j} \cdot T C+\sum_{j} \sum_{a} \sum_{h} q_{h a j}^{2} \cdot c_{h a}^{2} \cdot G_{j} \cdot T C \\
& +\sum_{j} \sum_{b} \sum_{h} q_{h b j}^{3} \cdot c_{h b}^{3} \cdot G_{j} \cdot T C+\sum_{j} \sum_{e} \sum_{h} q_{h e j}^{4} \cdot c_{h e}^{4} \cdot G_{j} \cdot T C+\sum_{j} \sum_{d} \sum_{h} q_{h d j}^{5} \cdot c_{h d}^{5} \cdot G_{j} \cdot T C
\end{aligned}
$$

If $S(\eta)=\varnothing$, some of the phones in collection points are not recycled due to the limitations of logistics centers or disassembling and processing centers. As a consequence, we define a penalty function

$$
C\left(X^{0}, \mathrm{X}^{1} \mid \eta\right)=\max _{s \in S^{\prime}(\eta)} Z \text { and } S^{\prime}(\eta)=\left\{q_{i l j}^{0}, q_{l h j}^{1}, q_{h a j}^{2}, q_{h b j}^{3}, q_{h e j}^{4}, q_{h d j}^{5} \mid \text { s.t.(1) (7),(11) }\right\} \text {. }
$$

Then we set up a stochastic programming model (16) to minimize expected cost:

$$
\begin{aligned}
& \min _{X^{0}, X^{1}} \int_{0}^{\infty} \operatorname{Pr}\left\{\eta \in \Omega \mid C\left(X^{0}, X^{1} \mid \eta\right) \geq q\right\} d q \\
& \text { s.t.(10),(11),(13) }
\end{aligned}
$$

In summary, an uncertain function is simulated first to tackle the above model:

$$
U:\left(X^{0}, X^{1}\right) \rightarrow \min _{X^{0}, X^{1}} \int_{0}^{\infty} \operatorname{Pr}\left\{\eta \in \Omega \mid C\left(X^{0}, X^{1} \mid \eta\right) \geq q\right\} d q
$$

Then the steps are as follows:

Step $1 \operatorname{Set} U\left(X^{0}, X^{1}\right)=0$;

Step2 Generate the realized value from sample space $\Omega$ according to probability measure $\operatorname{Pr}$;

Step3 Solve the linear programming problem and get the objective function value $Z$;

Step4 $U\left(X^{0}, X^{1}\right)=U\left(X^{0}, X^{1}\right)+Z$;

Step5 Repeat Step2 $\sim$ Step 4 for $N$ times ( $N$ is big enough);

Step6 Return the value $U\left(X^{0}, X^{1}\right) / N$.

\subsection{Model Solving}

We use genetic algorithm when solving the above stochastic programming model. For this facility selection problem, we adopt a binary encoding. The first $L$ digits stand for selection strategies of logistics centers and the rest $H$ digits are for disassembling and processing centers. The concrete steps are as follows:

Step 1 Set parameters: length of chromosome $N$, times of stochastic simulation $M$, maximal iterations $G E N$, population size $P o p-s i z e$, crossover probability $P_{c}$, mutation probability $P_{m}$; 
Step 2 Generate pop - size chromosomes randomly,

$$
V_{k}=\left(X^{0}, X^{1}\right), k=1,2, \cdots, \text { pop }- \text { size } ;
$$

Step3 Calculate the objective function value of each chromosome according to 4.7;

Step4 Define evaluation function based on order:

$$
\lambda(1-\lambda)^{k-1}, k=1,2, \cdots, \text { pop }- \text { size }, \lambda \in(0,1) \text {. Sort the chromosomes using such }
$$
function.

Step5 Use the roulette wheel method to select chromosomes for new population;

Step6 Use the two-point crossover method to update chromosomes;

Step 7 Adopt the basic bit mutation strategy to update chromosomes;

Step8 Repeat Step3 Step7 until the maximal iterations;

Step9 Return the best $V_{k}^{*}$ as the final results.

\section{Numerical Simulation and Suggestions}

Consider a third party reverse logistics enterprise that plan to start recycling mobile phones and construct a reverse logistics network. The data involved are product data and logistics data. Because few enterprises try to open such business in China, coupled with the fact that enterprises keep related data secret, it is inconvenient and difficult to conduct empirical studies and obtain data. In order to increase data authenticity and achieve our goal of testing the validity and utility of the model, the product data of the following example are gathered from data of main cell phone brands in the market. And the logistics data are acquired based on data analysis and extraction of related literature and researches [17, 19, 23]. In this example, there are 2 types of cell phones with weights of $110 \mathrm{~g}$ and $100 \mathrm{~g}$ respectively. There are 4 existing collection points, 2 existing parts manufactories, 2 existing raw material processing plants, 2 existing secondary markets, 2 existing waste disposal sites, 5 alternative logistics centers and 2 alternative disassembling and processing centers. Unit transportation cost is $0.001 / \mathrm{g} . \mathrm{km}$. The values of $\alpha_{1 j}$, $\alpha_{2 j}, \alpha_{3 j}, \alpha_{4 j}$ for the first products are $0.08,0.52,0.21$ and 0.19 , and the values for the second are 0.11, 0.51, 0.20 and 0.18. $\gamma=1$ and $\beta=100$. The number limitations of logistics centers and disassembling and processing centers are 3 and 2 respectively. Other parameters are showed in Table 1 to Table 4(LC is short for logistics center; DPC is short for disassembling and processing center; CP is short for collection point; PM is short for parts manufactory; RM is short for raw material processing plant; SM is short for secondary market; WDS is short for waste disposal site)

Table 1. Unit Operating Cost

\begin{tabular}{ccccccccc}
\hline & LC1 & LC2 & LC3 & LC4 & LC5 & DPC1 & DPC2 & DPC3 \\
\hline Product1 & 0.2 & 0.15 & 0.18 & 0.13 & 0.23 & 0.3 & 0.28 & 0.32 \\
Product2 & 0.18 & 0.14 & 0.15 & 0.1 & 0.2 & 0.28 & 0.27 & 0.3 \\
\hline
\end{tabular}

Table 2. Fixed Asset Investment Cost and Capacity Limitation

\begin{tabular}{ccccccccc}
\hline & LC1 & LC2 & LC3 & LC4 & LC5 & DPC1 & DPC2 & DPC3 \\
\hline $\begin{array}{c}\text { Fixed } \\
\text { Cost }\end{array}$ & 125000 & 165000 & 100000 & 195000 & 150000 & 350000 & 400000 & 370000
\end{tabular}


Table 3. Distribution Function of Recycling Quantity

\begin{tabular}{ccccc}
\hline & CP1 & CP2 & CP3 & CP4 \\
\hline Product1 & $U[8000,10000]$ & $U[6000,7000]$ & $U[12000,14000]$ & $U[10000,11000]$ \\
Product2 & $U[7000,10000]$ & $U[6000,8000]$ & $U[12000,14000]$ & $U[9000,10000]$ \\
\hline
\end{tabular}

Table 4. (1) Distance between Related Facilities (km)

\begin{tabular}{cccccccc}
\hline & CP1 & CP2 & CP3 & CP4 & DPC1 & DPC2 & DPC3 \\
\hline LC1 & 12 & 15 & 13 & 18 & 35 & 30 & 28 \\
LC2 & 14 & 10 & 17 & 16 & 32 & 37 & 35 \\
LC3 & 16 & 15 & 12 & 14 & 28 & 31 & 36 \\
LC4 & 17 & 16 & 11 & 13 & 31 & 27 & 30 \\
LC5 & 15 & 14 & 19 & 11 & 30 & 29 & 32 \\
\hline
\end{tabular}

Table 4. (2) Distance between Related Facilities (km)

\begin{tabular}{ccccccccccccc}
\hline & CP1 & CP2 & CP3 & CP4 & PM1 & PM2 & RM1 & RM2 & SM1 & SM2 & WDS1 & WDS2 \\
\hline DPC1 & 40 & 42 & 38 & 40 & 17 & 18 & 22 & 20 & 30 & 28 & 15 & 14 \\
DPC2 & 35 & 40 & 40 & 39 & 17 & 16 & 23 & 22 & 28 & 27 & 16 & 14 \\
DPC3 & 42 & 46 & 37 & 38 & 15 & 19 & 20 & 25 & 25 & 26 & 13 & 15 \\
\hline
\end{tabular}

\subsection{Results}

We use Matlab7.0 to compile the above hybrid intelligent algorithm program and related parameters are set up as follows:

$$
M=100, G E N=200, \text { Pop }- \text { size }=30, P_{c}=0.7, P_{m}=0.3, \lambda=0.05 .
$$

We used 4 hours and got the optimal objective function value $2.2574 \times 10^{6}$ in the $104^{\text {th }}$ iteration. Table 5 displays the results. That is to say, selecting the first logistics center, the third logistics center, the first disassembling and processing center and the third disassembling and processing center.

Table 5. Facility Selection Results

\begin{tabular}{cccccccc}
\hline & \multicolumn{3}{c}{ Logistics Center } & \multicolumn{3}{c}{ Disassembling and Processing Center } \\
\hline 1 & 2 & 3 & 4 & 5 & 1 & 2 & 3 \\
1 & 0 & 1 & 0 & 0 & 1 & 0 & 1 \\
\hline
\end{tabular}

\subsection{Parameter Sensitivity Analysis}

Then we carry out multi-factor sensitivity analysis of parameters (Table 6), namely GEN , Pop-size, $P_{c}, P_{m}, \lambda$ to see if the selection of each parameter could have certain effects on the results.

Table 6. Results of Sensitivity Analysis

\begin{tabular}{ccccccc}
\hline GEN & Pop-size & $P_{c}$ & $P_{m}$ & $\lambda$ & Results & Optimal Value (Generation) \\
\hline 200 & 30 & 0.6 & 0.2 & 0.05 & 10100101 & $2.2556 \times 10^{6}(176$ th $)$
\end{tabular}




$\begin{array}{lllllll}250 & 30 & 0.8 & 0.1 & 0.07 & 10100101 & 2.2574 \times 10^{6}(116 \text { th }) \\ 200 & 20 & 0.6 & 0.3 & 0.07 & 10100101 & 2.2581 \times 10^{6}(174 \text { th }) \\ 200 & 30 & 0.7 & 0.3 & 0.08 & 10100101 & 2.2559 \times 10^{6}(116 \text { th }) \\ 250 & 20 & 0.7 & 0.2 & 0.05 & 10100101 & 2.2576 \times 10^{6}(83 \text { th })\end{array}$

From Table 6 , we can come to a conclusion that the consequences of facility selection are the same (logistics center 1 and 3; disassembling and processing center 1 and 3). This algorithm has good stability and applicability and can be used when solving such problem.

\subsection{Suggestions}

Though some methods have been proposed related to the recycling of cell phones, the state quote of political and economic environment of China for reverse logistics network is still not that optimistic. The Chinese government has set up several laws to ensure the efficiency of recycling for electronic products, including TV sets, air conditioner, computers, fridges and washing machines except mobile phones. As a result, from the perspective of government, it should complete the legislation for cell phones and strengthen the supervision of the market. Additionally, capital investment and politics support are necessary. Last but not least, it has to perfect the market construction which can provide a convenient channel for users to put their waste phones to the collection points.

In terms of the third party enterprises, they should choose locations of facilities reasonably and accelerate the pace of facility construction, asset allocation and personnel training included, which can in turn ensure the quality of warehousing and transport services and the correctness and rationality of the following process. The network capacity should be in coordination with the volume to be processed and the enterprises themselves should perfectly cooperate with other players of the supply chain. They should not only consider the cost of the construction, but also build the network for the sake of the whole society.

\section{Summary}

The recycling and reuse of old mobile phones is a significant problem that needed to be solved in China. And such recycling project should take advantages of third party reverse logistics. At the same time, what really counts for a third party reverse logistics enterprise is the design of its network. So in this paper, we completely analyze the recycling network structure and design the whole procedure first. Then a programming model is proposed with objectives of minimizing cost and environmental effects. To solve the layout of key facilities, a hybrid intelligent algorithm is put forward. Finally we test the validity of the model and the algorithm by displaying an example.

As for mobile phone industry, the process of recycling is considered completely compared with formal literature and a network is established according to the whole process. This paper includes the following creative points. First of all, the model involves several types of products and takes uncertainty of quantity into account. Secondly, environment objective are brought in coupled with cost objective occurred generally in former literature to ensure objective function. Most importantly, we design a hybrid intelligent algorithm to solve the problem of programming in random environment which enhances the accuracy and validity of the results. From the consequences derived from the model and sensitivity analysis, we conclude that this model can be used to solve the design problem of the third party reverse logistics network for cell phones. What's more, this algorithm processes good stability and can be applied for guiding practice. 


\section{Acknowledgments}

This paper is supported by Natural Science Foundation of China (Grant No.713900334), EC-China Research Network on Integrated Container Supply Chains (Grant No.612546), Program for New Century Excellent Talents in University (Grant No.110567) and the Lab of Logistics Management and Technology of Beijing Jiaotong University.

\section{References}

[1] J. P. Guiltinan and N. G. Nwokoye, "Developing distribution channels and systems in the emerging recycling industries", International Journal of Physical Distribution \& Logistics Management, vol. 6, no. 1, (1975), pp. 28-38.

[2] P. M. Ginter and J. M. Starling, "Reverse distribution channels for recycling", California Management Review, vol. 20, no. 3, (1978).

[3] R. Batta and S. S. Chiu, "Optimal obnoxious paths on a network: transportation of hazardous materials", Operations Research, vol. 36, no. 1, (1988), pp. 84-92.

[4] V. Jayaraman, V. D. R. Guide Jr. and R. Srivastava, "A closed-loop logistics model for remanufacturing", Journal of the Operational Research Society, (1999), pp. 497-508.

[5] M. Fleischmann, J. M. Bloemhof-Ruwaard, R. Dekker, E. Van der Laan, J. A. Van Nunen and L. N. Van Wassenhove, "Quantitative models for reverse logistics: a review", European Journal of Operational Research, vol. 103, no. 1, (1997), pp. 1-17.

[6] J. Bautista and J. Pereira, "Modeling the problem of locating collection areas for urban waste management", An application to the metropolitan area of Barcelona. Omega, vol. 34, no. 6, (2006), pp. 617-629.

[7] I. M. Langella, "Heuristics for demand-driven disassembly planning", Computers \& Operations Research, vol. 34, no. 2, (2007), 552-577.

[8] R. K. Pati, P. Vrat and P. Kumar, "A goal programming model for paper recycling system”, Omega, vol. 36, no. 3, (2008), pp. 405-417.

[9] S. A. Alumur, S. Nickel, F. Saldanha-da-Gama and V. Verter, "Multi-period reverse logistics network design", European Journal of Operational Research, vol. 220, no. 1, (2012), pp. 67-78.

[10] M. Eskandarpour, S. H. Zegordi and E. Nikbakhsh, "A parallel variable neighborhood search for the multi-objective sustainable post-sales network design problem”, International Journal of Production Economics, vol. 145, no. 1, (2013), pp. 117-131.

[11] C. Harris and K. B. Martin, "The reverse logistics of online retailing, its evolution and future directions", Journal of System and Management Sciences, vol. 4, no. 2, (2014), pp. 1-14.

[12] Q. Y. Yun, "Research on value of third-party reverse logistics. Service Systems and Service Management (ICSSSM)", 7th International Conference on. IEEE, (2000), pp. 1-4.

[13] K. Govindan, M. Palaniappan, Q. Zhu and D. Kannan, "Analysis of third party reverse logistics provider using interpretive structural modeling", International Journal of Production Economics, vol. 140, no. 1, (2012), pp. 204-211.

[14] P. Mou, D. Xiang and G. H. Duan, "Modeling and case study of the regional e-waste reverse logistics system", Sustainable Systems and Technology, ISSST"09, IEEE International Symposium on. IEEE, (2009), pp. 1-1.

[15] D. L. Hou, J. Q. Nie and L. L. Zou, "The selection of reverse logistics modes for used cell phone products", Industrial Engineering Journal, vol. 1, (2012), pp. 011.

[16] P. Golinska and A. Kawa, "Remanufacturing in automotive industry: Challenges and limitations", Journal of Industrial Engineering and Management, vol. 4, no. 3, (2011), pp. 453-466.

[17] Y. Dai and Z. J. Ma, Editor, "Recycling system and management mechanism of waste appliances", Science Press. China, (2010).

[18] J. Ashayeri and G. Tuzkaya, "Design of demand driven return supply chain for high-tech products", Journal of Industrial Engineering and Management, vol. 4, no. 3, (2011), pp. 481-503.

[19] Y. Liu, "Model of multi-objective closed-loop logistics network design for remanufacturing products", Science and Technology Management Research, vol. 5, (2009), pp. 299-301.

[20] K. Talluri and G. V. Ryzin, "A randomized linear programming method for computing network bid prices", Transportation Science, vol. 33, no. 2, (1999), pp. 207-216.

[21] A. Madansky, "Inequalities for stochastic linear programming problems", Management science, vol. 6, no. 2, (1960), pp. 197-204.

[22] S. Sen and J. L. Higle, “An introductory tutorial on stochastic linear programming models", Interfaces, vol. 29, no. 2, (1999), pp. 33-61.

[23] J. Xu, H. Yue and Y. H. Wang, Editor, "Decisions making and network constructions of reverse logistics system”, Posts \& Telecom Press. China, (2007). 